\title{
ON CLASSES OF FINITE RINGS
}

\author{
ALEKSANDR TSAREV
}

\begin{abstract}
A class of rings is a formation whenever it contains all homomorphic images of its members and if it is subdirect product closed. In the present paper, it is shown that the lattice of all formations of finite rings is algebraic and modular. Let $R$ be a finite commutative ring with an identity element. It is established that there is a one-to-one correspondence between the set of all invariant fuzzy prime ideals of $R$ and the set of all fuzzy prime ideals of each ring of the formation generated by $R$.
\end{abstract}

\section{INTRODUCTION}

The concept of formation first appeared in the 1960s in connection with finite solvable groups [13. Further research showed that formations are of general algebraic nature and can be applied to the study of not necessarily solvable finite and infinite groups, Lie algebras, universal algebras and even of a general algebraic system [20]. A well-known result in group theory states that any formation of finite groups is saturated iff it is local (see Theorem 4.6 in the book [12]). In contrast to the group case, not every saturated formation of Lie and Leibniz algebras, rings, etc. can be locally defined. However, these formations have found various applications. Consider some examples.

Example 1.1 (Formations of monoids). There is a bijective correspondence between formations of finite monoids and the formations of languages. This result enables to study classes of regular languages which do not form varieties of languages [2]. Theorem 6 of the paper [3] confirms the existence of a bijective correspondence between formations of monoids and formations of congruences. This result does not require finiteness on monoids nor finite-index conditions on congruences. Languages associated with saturated formations of groups are described in [4], and in [22] it was shown that the mentioned result is applicable to the languages corresponding to $\tau$-closed saturated formations of finite groups.

2010 Mathematics Subject Classification. Primary 20D10; Secondary 16D99, 06B23, 03E72.

Key words and phrases. Finite ring; Formation; Lattice of formations; Algebraic lattice; Modular lattice; Fuzzy set; Fuzzy ideal.

This research was supported by Basic Science Research Program to RIBS of Jeju National University through the National Research Foundation of Korea (NRF) funded by the Ministry of Education (No. 2019R1A6A1A10072987) and by grant F19RM-071 from the Belarusian Republican Foundation for Fundamental Research. 
Example 1.2 (Formations of monounary algebras). The lattice of all formations of finite monounary algebras is isomorphic to the lattice of all hereditary subsets of a certain poset [16. The lattice of all formations of finite monounary algebras is distributive, but for the lattice of formations of at most countable monounary algebras this is not true; see [19].

Example 1.3 (Formations of lattice ordered groups). Jakubík [15] proved that the set of all formations of lattice ordered groups is a complete Brouwerian lattice, and the set of all formations of GMV-algebras is isomorphic to a principal ideal of the lattice of all formations of lattice ordered groups.

Example 1.4 (Formations of solvable Lie and Leibniz algebras). The theory of saturated formations of solvable Lie algebras is set out in Barnes and GastineauHills [5], and Barnes [6]. Over a field of nonzero characteristic, a saturated formation of solvable Lie algebras has at most one local definition, but a locally defined saturated formation of solvable Leibniz algebras other than that of nilpotent algebras has more than one local definition [7].

Example 1.5 (Formations of multirings). Christensen [10] showed that there exist Frattini closed formations of finite rings that are not local. Shemetkov [20] introduced the concept of formations of multirings, a special case of which is formations of finite rings. In the book [20] we can find various examples of applications of these formations, as well as discussion of related problems.

Question [20, Problem 3.51]. Is it true that any one-generated n-multiply local formation of rings has only a finite set of $n$-multiply local subformations?

Problem [20, Problem 22.8]. Describe finite non-one-generated formations of rings for which all proper subformations are one-generated.

This short review gives the motivation to study formations of finite rings.

\section{Statement of the Result}

Formations of finite rings were first introduced in [10] in order to obtain an analog of Lubeseder's theorem. We prove the following theorem.

Theorem 2.1. The lattice of all formations of finite rings is algebraic and modular.

An analogous result is already known for formations of finite groups [23, 21]. However, several ideas from the theory of formations of finite groups are of a universal nature, and can be used in the investigations of various algebraic systems. The term formation used in the theorem has a natural definition analogous to that used in group theory (see Chapter II of the book [12]).

A ring theoretical class or class of rings $\mathfrak{X}$ is a set of rings with the property that if $R \in \mathfrak{X}$, then every ring isomorphic to $R$ belongs to $\mathfrak{X}$. We refer to a class of rings as a homomorph whenever it contains all homomorphic images of its members and as a formation if in addition it is subdirect product closed; i.e., a formation is a 
class of finite rings $\mathfrak{F}$ which is both $\mathrm{Q}$-closed and $\mathrm{R}_{0}$-closed in the sense of [20] or [12, 1, 9].

The smallest formation of finite rings containing a class of finite rings $\mathfrak{X}$ is $\mathrm{QR}_{0} \mathfrak{X}$, composed of all rings that can be expressed as quotients of subdirect products of a finite number of rings in $\mathfrak{X}$. When $\mathfrak{X}=(R)$ consists only of the rings isomorphic to $R$, we obtain that the smallest formation containing $R$ is $\mathrm{QR}_{0}(R)$; such a formation is called one-generated. Hence a class of finite rings $\mathfrak{F}$ is a formation iff $\mathfrak{F}=\mathrm{QR}_{0} \mathfrak{F}$.

In the scope of groups, formations generalize some notions as solvability, supersolvability and nilpotency of groups. Let us consider an example for formations of rings.

Example 2.2 (Locally defined formations of finite rings [10]). For any ring $R$, the intersection $\Phi(R)$ of its maximal ideals, when such exist, is called the Frattini subring of $R$. For finite rings $\Phi(R)$ is contained in the Jacobson radical $J(R)$ of $R$. We are concerned with classes of rings that contain a ring $R$ whenever they contain its Frattini factor ring $R / \Phi(R)$. Such classes are said to be Frattini closed.

One of the most elementary nontrivial examples of a Frattini closed formation of rings is the class $\mathfrak{N}$ of all finite nilpotent rings. This class can be described locally in the sense that $R \in \mathfrak{N}$ iff the minimal ideals of its factor rings $R / K$ are trivial left $R$-modules.

Following [10] we refer to the minimal ideals of the factor rings of a finite ring $R$ as chief factors of $R$. Since each chief factor has prime characteristic it can be classified, according to which prime $p$ is involved, as a $p$-chief factor. Denote for any chief factor $H / K$ of $R$ its left annihilator $\{r \mid r \in R$ and $r H \subseteq K\}$ in $R$ by $A_{R}(H / K)$. Given a set of primes $\pi$ and a function $f$ with domain $\pi$ whose images are formations of finite rings, the class $\mathfrak{F}$ of $\pi$-rings whose $p$-chief factors $H / K$ have the property

$$
R / A_{R}(H / K) \in f(p) \text { for each } p \in \pi
$$

is a formation. Such a formation is called the local formation defined by the formation function $f$ with support $\pi$.

In view of the primary decomposition of finite rings, we see that for any $p \in \pi$, the class $\mathfrak{F}_{p}$ of $p$-rings in $\mathfrak{F}$ is a formation and is defined locally by the formation function $f_{p}$ with support $\{p\}$ and image $\{f(p)\}$. The most elementary nontrivial local formations are the formations $\mathfrak{N}_{p}$ of finite nilpotent $p$-rings in the sense that they contain no proper local formations.

Problem. Describe algebraic and modular lattices of local formations of finite rings.

\section{LATtice theOREtical PRELIMINARIES}

Let $\Theta$ be a set of formations. A formation in $\Theta$ is called a $\Theta$-formation. If the intersection of every set of $\Theta$-formations belongs to $\Theta$ and there is a $\Theta$-formation $\mathfrak{F}$ such that $\mathfrak{M} \subseteq \mathfrak{F}$ for every other $\Theta$-formation $\mathfrak{M}$, then $\Theta$ is called a complete lattice of formations. Any complete lattice of formations is a complete lattice in the 
ordinary sense. We note that $\emptyset$ and $(0)$ are formations and the set of all formations of finite rings is the complete lattice of formations.

Let $\Theta$ be a complete lattice of formations of finite rings. If $\mathfrak{M}, \mathfrak{H} \in \Theta$, then $\mathfrak{M} \cap \mathfrak{H}$ is the greatest lower bound for $\{\mathfrak{M}, \mathfrak{H}\}$ in $\Theta$; and $\mathrm{QR}_{0}(\mathfrak{M} \cup \mathfrak{H})$ is the least upper bound for $\{\mathfrak{M}, \mathfrak{H}\}$ in $\Theta$.

An element $a$ of a lattice $\Theta$ is compact [8] if $a \leqslant \vee\left(x_{j} \mid j \in S\right)$ holds for $a \leqslant \vee\left(x_{j} \mid j \in J\right)$ and some finite subset $S \subset J$. A complete lattice is called algebraic if each element of it is the union (i.e., the least upper bound) of some set of compact elements.

\section{THE PROOF}

All rings considered are finite. The notation $J \triangleleft R$ means that $J$ is an ideal of a ring $R$, and we use the notation $R / I$ for a quotient ring of $R$ modulo $I$ if $I \triangleleft R$. We observe that a class of rings $\mathfrak{F}$ is a formation iff it satisfies the following two conditions:

(1) if $R \in \mathfrak{F}$ and $J \triangleleft R$, then $R / J \in \mathfrak{F}$; and

(2) if $R / I_{1}, R / I_{2} \in \mathfrak{F}$, then $R / I_{1} \cap I_{2} \in \mathfrak{F}$ for any $I_{1}, I_{2} \triangleleft R$.

Proof of Theorem 2.1. STEP 1 (ALGEBRAICITy). We show first that each onegenerated formation $\mathfrak{F}=\mathrm{QR}_{0}(R)$ is a compact element in the lattice of all formations of rings.

Let $\mathfrak{F} \subseteq \mathrm{QR}_{0}\left(\bigcup_{i \in I} \mathfrak{F}_{i}\right)$, where $\left\{\mathfrak{F}_{i} \mid i \in I\right\}$ is a set of formations. Then $R \in$ $\mathrm{QR}_{0}\left(\bigcup_{i \in I} \mathfrak{F}_{i}\right)$. Hence $R \simeq T / J$, where $J \triangleleft T \in \mathrm{R}_{0}\left(\cup_{i \in I} \mathfrak{F}_{i}\right)$. Then there are some $J_{k} \triangleleft T(k=1, \ldots, r)$ such that $T / J_{k} \in \cup_{i \in I} \mathfrak{F}_{i}$ and $J_{1} \cap \cdots \cap J_{r}=\{0\}$. Consequently, $T / J_{1} \in \mathfrak{F}_{i_{1}}, \ldots, T / J_{r} \in \mathfrak{F}_{i_{r}}$ for some $i_{1}, \ldots, i_{r} \in I$.

Thus for any $k \in\{1, \ldots, r\}$, we have $T / J_{k} \in \mathfrak{F}_{i_{1}} \cup \cdots \cup \mathfrak{F}_{i_{r}}$. Therefore $T \in$ $\mathrm{R}_{0}\left(\mathfrak{F}_{i_{1}} \cup \cdots \cup \mathfrak{F}_{i_{r}}\right)$. From $R \simeq T / J$ and $J \triangleleft T$, we have $R \in \mathrm{QR}_{0}\left(\mathfrak{F}_{i_{1}} \cup \cdots \cup \mathfrak{F}_{i_{r}}\right)$, and then

$$
\mathfrak{F}=\mathrm{QR}_{0}(R) \subseteq \mathrm{QR}_{0}\left(\mathfrak{F}_{i_{1}} \cup \cdots \cup \mathfrak{F}_{i_{r}}\right) .
$$

We show next that any nonempty formation of rings $\mathfrak{M}$ is the union (in the lattice of all formations of rings) of its one-generated subformations $\mathfrak{M}_{l}=\mathrm{QR}_{0}\left(R_{l}\right)$, where $l \in L$. Let $\mathfrak{Y}=\mathrm{QR}_{0}\left(\cup_{l \in L} \mathfrak{M}_{l}\right)$. We show now that $\mathfrak{M}=\mathfrak{Y}$. Let $R \in \mathfrak{M}$. Then

$$
R \in \mathrm{QR}_{0}(R) \subseteq \bigcup_{i \in L} \mathfrak{M}_{i} \subseteq \mathrm{QR}_{0}\left(\bigcup_{i \in L} \mathfrak{M}_{i}\right)=\mathfrak{Y} .
$$

Consequently, $\mathfrak{M} \subseteq \mathfrak{Y}$. The inverse inclusion is obvious; $\mathfrak{M}_{i} \subseteq \mathfrak{M}$ implies $\cup_{i \in L} \mathfrak{M}_{i} \subseteq$ $\mathfrak{M}$, and, consequently, $\mathfrak{Y} \subseteq \mathfrak{M}$.

Step 2 (MODUlarity). We wish to show that the following equality holds, for any formations of rings $\mathfrak{X} \subseteq \mathfrak{Y}$ and $\mathfrak{F}$ :

$$
\mathfrak{Y} \cap \mathrm{QR}_{0}(\mathfrak{X} \cup \mathfrak{F})=\mathrm{QR}_{0}(\mathfrak{X} \cup(\mathfrak{Y} \cap \mathfrak{F})) \text {. }
$$

The inclusion " $\supseteq$ " is trivial. Let $A \in \mathfrak{Y} \cap \mathrm{QR}_{0}(\mathfrak{X} \cup \mathfrak{F})$. Then $A$ is a homomorphic image of some ring $R \in \mathrm{R}_{0}(\mathfrak{X} \cup \mathfrak{F})$, and we can find some ideals $J_{1}$ and $J_{2}$ of the $\operatorname{ring} R$ such that $R / J_{1} \in \mathfrak{X}$ and $R / J_{2} \in \mathfrak{F}$ with $J_{1} \cap J_{2}=\{0\}$. 
Let $A \cong R / I$, where $I \triangleleft R$. It is well known that the set of all ideals of a ring forms a complete modular lattice with respect to set inclusion. Thus, by modular law, we have $J_{1} \cap\left(\left(J_{1} \cap I\right)+J_{2}\right)=\left(J_{1} \cap I\right)+\left(J_{1} \cap J_{2}\right)=J_{1} \cap I$. We note that

$$
\begin{gathered}
\left(R /\left(J_{1} \cap I\right)\right) /\left(J_{1} /\left(J_{1} \cap I\right)\right) \cong R / J_{1} \in \mathfrak{X}, \text { and } \\
\left(R /\left(J_{1} \cap I\right)\right) /\left(\left(J_{1} \cap I\right)+J_{2} /\left(J_{1} \cap I\right)\right) \cong R /\left(J_{1} \cap I\right)+J_{2} \in \mathfrak{F} .
\end{gathered}
$$

Hence, $R /\left(J_{1} \cap I\right) \in \mathrm{R}_{0}(\mathfrak{X} \cup \mathfrak{F})$. From $R / I \in \mathfrak{Y}$ and $\mathfrak{X} \subseteq \mathfrak{Y}$, we conclude that $R /\left(J_{1} \cap I\right) \in \mathfrak{Y}$.

Consequently, $R /\left(J_{1} \cap I\right) \in \mathrm{R}_{0}(\mathfrak{X} \cup(\mathfrak{Y} \cap \mathfrak{F}))$ implies $A \in \mathrm{QR}_{0}(\mathfrak{X} \cup(\mathfrak{Y} \cap \mathfrak{F}))$. This proves the theorem.

Let $\mathfrak{F}$ and $\mathfrak{H}$ be formations such that $\mathfrak{H} \subseteq \mathfrak{F}$. We denote by $\mathfrak{F} / \mathfrak{H}$ the lattice of all formations $\mathfrak{M}$ such that $\mathfrak{H} \subseteq \mathfrak{M} \subseteq \mathfrak{F}$. As an immediate corollary from the modularity of the lattice of all formations of rings, we obtain the following result.

Corollary 4.1. For any two formations $\mathfrak{M}$ and $\mathfrak{F}$ the lattices $\mathrm{QR}_{0}(\mathfrak{M} \cup \mathfrak{F}) / \mathfrak{M}$ and $\mathfrak{F} /(\mathfrak{F} \cap \mathfrak{M})$ are isomorphic.

\section{LATtice FEATURES}

Let $\mathfrak{F}$ be a nonempty class of rings, and $J \triangleleft R$. We say that $J$ is the $\mathfrak{F}$-residual of $R$ if $R / J \in \mathfrak{F}$, and $R / I \in \mathfrak{F}$ always implies $J \subseteq I$. If a ring $R$ possesses the $\mathfrak{F}$-residual then we denote it by $R^{\mathfrak{F}}=\bigcap\{J \triangleleft R \mid R / J \in \mathfrak{F}\}$. Let $\mathfrak{X}$ be a class of finite rings, and $\mathfrak{Y}$ be a formation of finite rings. We define the formation product of $\mathfrak{X}$ and $\mathfrak{Y}$ as follows:

$$
\mathfrak{X Y}=\left(R \mid R^{\mathfrak{Y}} \in \mathfrak{X}\right) .
$$

Proposition 5.1. Let $\mathfrak{X}$ and $\mathfrak{Y}$ be nonempty formations of finite rings. Then

(1) $\mathfrak{X Y Y}$ is a formation of finite rings;

(2) $R^{\mathfrak{X Y}}=\left(R^{\mathfrak{Y}}\right)^{\mathfrak{X}}$ for all finite rings $R$.

Proof. The proof of the assertion repeats the finite group case; see [12, p. 338].

Lemma 5.2. Let $\mathfrak{F}_{1}$ and $\mathfrak{F}_{2}$ be nonempty formations of rings and $A \in \mathrm{QR}_{0}\left(\mathfrak{F}_{1} \cup \mathfrak{F}_{2}\right)$. Then there exist rings $A_{1} \in \mathfrak{F}_{1}$ and $A_{1} \in \mathfrak{F}_{1}$ such that

$$
A \in \mathrm{QR}_{0}\left(\mathrm{QR}_{0}\left(A_{1}\right) \cup \mathrm{QR}_{0}\left(A_{2}\right)\right) .
$$

Proof. We see that $A \cong R / J$, where $R \in \mathrm{R}_{0}\left(\mathfrak{F}_{1} \cup \mathfrak{F}_{2}\right)$. Then $R$ possesses ideals $J_{1}$, $\ldots, J_{t}(t \geqslant 2)$ such that $\cap_{i=1}^{t} J_{i}=\{0\}$ and $R / J_{i} \in \mathfrak{F}_{1} \cup \mathfrak{F}_{2}$ for $i=1, \ldots, t$. We note that $R^{\mathfrak{F}_{1}} \cap R^{\mathfrak{F}_{2}}=\{0\}$. Hence $R \in \mathrm{R}_{0}\left(R / R^{\mathfrak{F}_{1}}, R / R^{\mathfrak{F}_{2}}\right)$. Set $A_{1}=R / R^{\mathfrak{F}_{1}}$ and $A_{2}=R / R^{\mathfrak{F}_{2}}$. We have finally

$$
A \cong R / J \in \mathrm{QR}_{0}\left(R / R^{\mathfrak{F}_{1}}, R / R^{\mathfrak{F}_{2}}\right)=\mathrm{QR}_{0}\left(\mathrm{QR}_{0}\left(A_{1}\right) \cup \mathrm{QR}_{0}\left(A_{2}\right)\right) \subseteq \mathrm{QR}_{0}\left(\mathfrak{F}_{1} \cup \mathfrak{F}_{2}\right) .
$$

The concept of a separated lattice of formations of finite groups was proposed in the book [21. We introduce a similar definition for formations of finite rings. Let $\mathfrak{X}$ be a nonempty class of rings. We say that a complete lattice of formations $\Theta$ is $\mathfrak{X}$-separated if for 
- any term $\xi\left(x_{1}, \ldots, x_{m}\right)$ of signature $\left\{\cap, \mathrm{QR}_{0}\right\}$,

- any $\Theta$-formations $\mathfrak{F}_{1}, \ldots, \mathfrak{F}_{m}$, and

- any ring $A \in \mathfrak{X} \cap \xi\left(\mathfrak{F}_{1}, \ldots, \mathfrak{F}_{m}\right)$,

there exist $\mathfrak{X}$-rings $A_{1} \in \mathfrak{F}_{1}, \ldots, A_{m} \in \mathfrak{F}_{m}$ such that $A \in \xi\left(\mathrm{QR}_{0}\left(A_{1}\right), \ldots, \mathrm{QR}_{0}\left(A_{m}\right)\right)$.

Denote by $\mathfrak{R}$ the class of all formations of finite rings.

Proposition 5.3. The lattice of all formations of finite rings formations is $\mathfrak{R}$ separated.

Proof. Let $\xi\left(x_{1}, \ldots, x_{m}\right)$ be a term of signature $\left\{\cap, \mathrm{QR}_{0}\right\}$, and $\mathfrak{F}_{1}, \ldots, \mathfrak{F}_{m}$ be formations of rings. Suppose that $A \in \xi\left(\mathfrak{F}_{1}, \ldots, \mathfrak{F}_{m}\right)$.

We proceed by induction on the number $r$ of occurences of the symbols in $\left\{\cap, \mathrm{QR}_{0}\right\}$ into the term $\xi$. We show that there exist rings $A_{i} \in \mathfrak{F}_{i}(i=1, \ldots, m)$ such that $A \in \xi\left(\mathrm{QR}_{0}\left(A_{1}\right), \ldots, \mathrm{QR}_{0}\left(A_{m}\right)\right)$.

Let $r=0$. It is clear that $A \in \mathrm{QR}_{0}(A)$.

Let $r=1$. Then we have only two cases: either $A \in \mathfrak{F}_{1} \cap \mathfrak{F}_{2}$ or $A \in \mathrm{QR}_{0}\left(\mathfrak{F}_{1} \cup \mathfrak{F}_{2}\right)$. In the first case, we have $A \in \mathrm{QR}_{0}(A) \cap \mathrm{QR}_{0}(A)$. In the second case, by Lemma 5.2 there exist rings $A_{1} \in \mathfrak{F}_{1}$ and $A_{2} \in \mathfrak{F}_{2}$ such that $A \in \mathrm{QR}_{0}\left(\mathrm{QR}_{0}\left(A_{1}\right) \cup \mathrm{QR}_{0}\left(A_{2}\right)\right)$. Thus, the assertion for $r=1$ is true.

Let a term $\xi$ have $r>1$ occurrences of the symbols in $\left\{\cap, \mathrm{QR}_{0}\right\}$. We suppose that the assertion holds for terms with less than $r$ occurrences. Assume that $\xi$ is of the form

$$
\xi_{1}\left(x_{i_{1}}, \ldots, x_{i_{a}}\right) \triangle \xi_{2}\left(x_{j_{1}}, \ldots, x_{j_{b}}\right),
$$

where $\triangle \in\left\{\cap, \mathrm{QR}_{0}\right\}$ and $\left\{x_{i_{1}}, \ldots, x_{i_{a}}\right\} \cup\left\{x_{j_{1}}, \ldots, x_{j_{b}}\right\}=\left\{x_{1}, \ldots, x_{m}\right\}$.

By $\mathfrak{H}_{1}$ we denote the formation $\xi_{1}\left(\mathfrak{F}_{i_{1}}, \ldots, \mathfrak{F}_{i_{a}}\right)$, and by $\mathfrak{H}_{2}$ we denote the formation $\xi_{2}\left(\mathfrak{F}_{j_{1}}, \ldots, \mathfrak{F}_{j_{b}}\right)$. There exist rings $A_{1} \in \mathfrak{H}_{1}$ and $A_{2} \in \mathfrak{H}_{2}$ such that

$$
A \in \mathrm{QR}_{0}\left(A_{1}\right) \triangle \mathrm{QR}_{0}\left(A_{2}\right) .
$$

On the other hand, by induction, there exist rings $B_{1}, \ldots, B_{a} ; C_{1}, \ldots, C_{b}$ such that $B_{k} \in \mathfrak{F}_{i_{k}}, C_{k} \in \mathfrak{F}_{j_{k}}$,

$$
A_{1} \in \xi_{1}\left(\mathrm{QR}_{0}\left(B_{1}\right), \ldots, \mathrm{QR}_{0}\left(B_{a}\right)\right) \text { and } A_{2} \in \xi_{2}\left(\mathrm{QR}_{0}\left(C_{1}\right), \ldots, \mathrm{QR}_{0}\left(C_{b}\right)\right) .
$$

Suppose that $x_{i_{1}}, \ldots, x_{i_{t}}$ are not contained in $\xi_{2}$, and $x_{i_{t+1}}, \ldots, x_{i_{a}}$ are contained in $\xi_{2}$. Let $D_{i_{k}}=B_{k}$ if $k<t+1, D_{i_{k}}=B_{k} \times C_{q}$, where $q$ satisfies $x_{i_{k}}=x_{j_{q}}$ for all $k \geqslant t+1 k \geqslant t+1$. Let $D_{j_{k}}=C_{k}$ if $x_{j_{k}} \notin\left\{x_{i_{t+1}}, \ldots, x_{i_{a}}\right\}$.

We denote by $\mathfrak{T}_{p}$ the formation of rings $\mathrm{QR}_{0}\left(D_{i_{p}}\right)$, and by $\mathfrak{X}_{c}$ we denote the formation $\mathrm{QR}_{0}\left(D_{j_{c}}\right), p=1, \ldots, a ; c=1, \ldots, b$.

It follows that $A_{1} \in \xi_{1}\left(\mathfrak{T}_{1}, \ldots, \mathfrak{T}_{a}\right)$, and $A_{2} \in \xi_{2}\left(\mathfrak{X}_{1}, \ldots, \mathfrak{X}_{b}\right)$. There exist formations $\mathfrak{H}_{1}, \ldots, \mathfrak{H}_{m}$ such that

$$
A \in \xi_{1}\left(\mathfrak{H}_{i_{1}}, \ldots, \mathfrak{H}_{i_{a}}\right) \triangle \xi_{2}\left(\mathfrak{H}_{j_{1}}, \ldots, \mathfrak{H}_{j_{b}}\right)=\xi\left(\mathfrak{H}_{1}, \ldots, \mathfrak{H}_{m}\right),
$$

where $\mathfrak{H}_{i}=\mathrm{QR}_{0}\left(K_{i}\right)$, and $K_{i} \in \mathfrak{F}_{i}$. This proves the assertion. 


\section{Some APPLICATIONS}

Fuzzy sets introduced by Zadeh [24] and Klaua [17] became applied in fields such as pattern recognition, machine learning and data mining [14, 25]. Focusing on the structure of ring, Liu [18] introduced and studied the notions of fuzzy subrings and fuzzy ideals, and showed that the images and preimages under onto homomorphisms of fuzzy ideals are fuzzy ideals. Many authors have developed the fuzzy ring theory, but we see that not all the results on rings can be fuzzified. For instance, Dixit, Kumar, and Ajmal [11] discussed the conditions under which a given fuzzy ideal can or cannot be expressed as a union of two proper fuzzy ideals.

Recall that a fuzzy subset of a set $X$ is a function from $X$ into the closed interval $[0,1]$. Let $X$ and $X^{\prime}$ be any two sets, and $f: X \rightarrow X^{\prime}$ be any function. A fuzzy subset $\mu$ of $X$ is called $f$-invariant if $f(x)=f(y)$ implies $\mu(x)=\mu(y)$, where $x, y \in X$.

Let '.' be a binary composition in a set $X$, and $\mu$ and $\mu^{\prime}$ be any two fuzzy subsets of $X$. The product $\mu \mu^{\prime}$ is defined by

$$
\mu \mu^{\prime}(z)= \begin{cases}\sup \left(\min \left\{\mu(x), \mu^{\prime}(y)\right\}\right), & \text { for } x, y \in X \text { and } z=x \cdot y \\ 0, & \text { if } z \text { is not expressible as } z=x \cdot y \\ & \text { for all } x, y \in X\end{cases}
$$

Clearly, $\mu \mu^{\prime}$ is a fuzzy subset of $X$.

A fuzzy subset $\mu$ of a ring $R$ is called a fuzzy ideal of $R$ if it has the following two properties:

(1) $\mu(x-y) \geqslant \min \{\mu(x), \mu(y)\}$ for any $x, y \in R$; and

(2) $\mu(x y) \geqslant \max \{\mu(x), \mu(y)\}$ for any $x, y \in R$.

In the sequel, by a ring we shall always mean a finite commutative ring with identity. A fuzzy ideal $\mu$ of a ring $R$ is called fuzzy prime if for any fuzzy ideals $\mu$ and $\mu^{\prime}$ of $R$, the condition $\mu \mu^{\prime} \subseteq \mu$ implies that either $\mu \subseteq \mu^{\prime}$ or $\mu^{\prime} \subseteq \mu$.

We shall write form $R$ instead of $\mathrm{QR}_{0}(R)$ for the formation generated by $R$.

Lemma 6.1. Let $R$ be a ring and $\mathfrak{F}=$ form $R$. Then the following two conditions hold:

(1) Any invariant fuzzy prime ideal of $R$ corresponds in a natural way to a fuzzy prime ideal of each member of $\mathfrak{F}$.

(2) Any fuzzy prime ideal of each member of $\mathfrak{F}$ corresponds in a natural way to a fuzzy prime ideal of $R$.

Proof. We note that the formation $\mathfrak{F}$ consists of all quotients of subdirect products of copies of $R$. Let $f$ be any homomorphism from the ring $R$ onto a ring $A \in$ form $R$. Then $f(R)=A$.

(1) Let $\mu$ be an $f$-invariant fuzzy prime ideal of $R$. Then by [11, Theorem 4.4], $f(\mu)$ (see [11, Lemma 4.1]) is a fuzzy prime ideal of $A$.

(2) Let $\nu$ be a fuzzy prime ideal of $A$. Then $f^{-1}(\nu)$ (see [11, Lemma 4.1]) is a fuzzy prime ideal of $R$ by [11, Theorem 4.5].

An immediate consequence of this lemma is the following result. 
Proposition 6.2. Let $R$ be a ring and $\mathfrak{F}=$ form $R$. Then there is a one-to-one correspondence between the set of all invariant fuzzy prime ideals of $R$ and the set of all fuzzy prime ideals of each ring of $\mathfrak{F}$.

Finally, we note that by Theorem 2.1 every formation of finite rings is the join of some one-generated formations.

\section{ACKNOWLEDGEMENTS}

The author thanks the anonymous referee for many insightful comments that have greatly contributed to this paper.

\section{REFERENCES}

[1] A. Ballester-Bolinches and L.M. Ezquerro, Classes of Finite Groups, Springer, Dordrecht, 2006. MR 2241927

[2] A. Ballester-Bolinches, J.-É. Pin, X. Soler-Escrivà, Formations of finite monoids and formal languages: Eilenberg's variety theorem revisited, Forum Math. 26 (2014), no. 6, 1737-1761. MR 3334045

[3] A. Ballester-Bolinches, E. Cosme-Llópez, R. Esteban-Romero, J.J.M.M. Rutten, Formations of monoids, congruences, and formal languages, Sci. Ann. Comput. Sci. 25 (2015), no. 2, 171-209. MR 3438712

[4] A. Ballester-Bolinches, J.-É. Pin, X. Soler-Escrivà, Languages associated with saturated formations of groups, Forum Math. 27 (2015), no. 3, 1471-1505. MR 3341483.

[5] D.W. Barnes, H.M. Gastineau-Hills, On the theory of soluble Lie algebras, Math. Z. 106 (1968), 343-354. MR 0232807.

[6] D.W. Barnes, Saturated formations of soluble Lie algebras in characteristic 0, Arch. Math. (Basel) 30 (1978), no. 5, 477-480. MR 0503158.

[7] D.W. Barnes, On locally defined formations of soluble Lie and Leibniz algebras, Bull. Aust. Math. Soc. 86 (2012), no. 2, 322-326. MR 2979992

[8] G.D. Birkhoff, Lattice Theory, American Mathematical Society Colloquium Publications, 25. American Mathematical Society, Providence, R.I., 1979. MR 1567177

[9] J.A. Cabrera, I. Gutiérrez García, Sobre clases de grupos finitos solubles, Matemáticas: Enseñanza Universitaria 12 (2004), no. 2, 53-68.

[10] C. Christensen, An analogue of Lubeseder's theorem for formations of finite rings, J. Aust. Math. Soc. 16 (1973), 375-378. MR 0344285.

[11] V.N. Dixit, R. Kumar, N. Ajmal, Fuzzy ideals and fuzzy prime ideals of a ring, Fuzzy Sets and Systems 44 (1991), no. 1, 127-138. MR 1133988

[12] K. Doerk, T. Hawkes, Finite Soluble Groups, Walter de Gruyter, Berlin, 1992. MR 1169099

[13] W. Gaschütz, Zur Theorie der endlichen auflösbaren Gruppen, Math. Z. 80 (1962/63), 300305. MR 179257

[14] E. Hüllermeier, Fuzzy methods in machine learning and data mining: Status and prospects, Fuzzy Sets and Systems 156 (2005), no. 3, 387-406. MR 2180474

[15] J. Jakubík, Formations of lattice ordered groups and of GMV-algebras, Math. Slovaca 58 (2008), no. 5, 521-534. MR 2434675

[16] D. Jakubíková-Studenovská, J. Pócs, Formations of finite monounary algebras, Algebra Universalis 68 (2012), no. 3-4, 249-255. MR 3029954

[17] D. Klaua, Über einen Ansatz zur mehrwertigen Mengenlehre, Monatsb. Deutsch. Akad. Wiss. Berlin 7 (1965), 859-867. MR 0193009

[18] W. Liu, Fuzzy invariant subgroups and fuzzy ideals, Fuzzy Sets and Systems 8 (1982), no. 2, 133-139. MR 0666626

[19] A.L. Rasstrigin, On lattices of formations of monounary algebras with finitely many cycles, Lobachevskii J. Math. 36 (2015), no. 4, 419-425. MR 3431203 
[20] L.A. Shemetkov, A.N. Skiba, Формации алгебраических систем [Formations of Algebraic Systems]. "Nauka", Moscow, 1989. MR 1007307

[21] A.N. Skiba, Алгебра формаций [Algebra of formations], Izdatel'stvo Belaruskaya Navuka, Minsk, 1997. MR 1659316

[22] A. Tsarev, Formations of finite monoids and their applications: formations of languages and $\tau$-closed saturated formations of finite groups, Ann. Univ. Ferrara Sez. VII Sci. Mat. 65 (2019), no. 2, 369-374. MR 4026430.

[23] N.N. Vorob'ev, Algebra of Classes of Finite Groups, P.M. Masherov Vitebsk State University, Vitebsk, 2012 (in Russian).

[24] L.A. Zadeh, Fuzzy sets, Information and Control 8 (1965), 338-353. MR 0219427

[25] Online Fuzzy Machine Learning and Data Mining, special issue of Inform. Sci. 220 (2013), 1-602.

Aleksandr Tsarev

Department of Mathematics, Jeju National University, Jeju 690-756, South Korea, and

Department of Mathematics \& IT, P.M. Masherov Vitebsk State University, 33 Moscow Av., 210038, Vitebsk, Belarus

alessandro.tsarev@yahoo.com

Received: October 10, 2018

Accepted: April 30, 2019 